\title{
Neutron Star Stability in Light of the Neutron Decay Anomaly
}

\author{
Benjamín Grinstein \\ Department of Physics, UC San Diego, La Jolla, California 92093, USA \\ Chris Kouvaris $^{\dagger}$ and Niklas Grønlund Nielsen ${ }^{\ddagger}$ \\ $C P^{3}$-Origins, University of Southern Denmark, Campusvej 55, DK-5230 Odense, Denmark
}

(Received 5 December 2018; published 28 August 2019)

\begin{abstract}
A recent proposal suggests that experimental discrepancies on the lifetime of neutrons can be resolved if neutrons decay to dark matter. At the same time it has been demonstrated that such a decay mode would soften the nuclear equation of the state resulting in neutron stars with a maximum mass much below currently observed ones. In this Letter, we demonstrate that appropriate dark matter-baryon interactions can accommodate neutron stars with mass above two solar masses. We compare this stabilization mechanism to one based on dark matter self-interactions, finding that it is less sensitive to the details of the nuclear equation of state. We present a simple microscopic model realization of this mechanism.
\end{abstract}

DOI: 10.1103/PhysRevLett.123.091601

Introduction.-A puzzling discrepancy between the neutron lifetime as measured in bottle and beam experiments has persisted for more than 20 years [1]. In bottle experiments, a number of neutrons $N$ are cooled down and stored in a container. At later times the remaining number of neutrons is counted and fit to a decaying exponential $\propto \exp \left(-t / \tau_{\text {bottle }}\right)$. In beam experiments, the rate of protons emitted from a beam of neutrons is fit to $d N / d t=-N / \tau_{\text {beam }}$. The current world average in the PDG [1] for the neutron lifetime in bottle experiments [2-6] is $\tau_{\text {bottle }}=879.6 \pm 0.6 \mathrm{~s}$, whereas the beam experiment measurements $[7,8]$ are significantly longer with a world average at $\tau_{\text {beam }}=888.0 \pm 2.0 \mathrm{~s}$. The discrepancy is currently at the $4 \sigma$ level.

Calculation of the neutron lifetime requires the nucleon axial coupling, $g_{A}$, as input. Both a global fit [9] and a recent $1 \%$ lattice-QCD determination [10] give $\tau_{n} \approx 885 \mathrm{~s}$. However, if only $g_{A}$ determinations dating from after 2002 are used, the resulting calculated lifetime agrees remarkably well with $\tau_{\text {bottle }}$ [11]. A resolution involving new physics is not out of the question, and an exciting proposal by Fornal and Grinstein [12] recently sparked significant interest in the subject. They suggested an invisible decay of the neutron into dark matter $(\mathrm{DM})$ in order to explain the neutron decay anomaly. Since the beam measurements are only sensitive to beta decay, one would expect $\tau_{\text {beam }}=\tau_{\text {bottle }} / \operatorname{Br}(n \rightarrow p+$ $\left.e+\bar{\nu}_{e}\right)$, suggesting that $\operatorname{Br}\left(n \rightarrow p+e+\bar{\nu}_{e}\right) \approx 99 \%$ with the remaining $1 \%$ branching into DM. Interestingly, the dark

Published by the American Physical Society under the terms of the Creative Commons Attribution 4.0 International license. Further distribution of this work must maintain attribution to the author(s) and the published article's title, journal citation, and DOI. Funded by SCOAP ${ }^{3}$. decay of the neutron hypothesis can be tested by visible decays of unstable nuclei [12]. A systematic analysis by Pfützner and Riisager shows that ${ }^{11} \mathrm{Be} \rightarrow{ }^{10} \mathrm{Be}$ and ${ }^{15} \mathrm{C} \rightarrow{ }^{14} \mathrm{C}$ are particularly sensitive tests [13], the former because the only irreducible background, from $\beta$-delayed $p$ emission, is reliably calculated to be 400 times smaller than observed [14], and the latter because there is no irreducible background and technology to measure minute amounts of ${ }^{14} \mathrm{C}$ is highly developed. Soon after the proposal in Ref. [12], it was realized that an invisible decay channel of the neutron would lead to partial conversion of neutrons to DM inside a neutron star (NS). In such a case the nuclear equation of state (EOS) softens so much at high densities that makes it impossible for a NS to support masses above $2 M_{\odot}$ [15-17]. This contradicts current observations setting the maximum NS mass above $\sim 2 M_{\odot}$.

The authors of Ref. [18] studied the NS stability including DM self-interactions mediated by dark photons, concluding that although such interactions provide enough pressure to support $2 M_{\odot} \mathrm{NSs}$, the DM particle that the neutron decay to must represent at best only a small fraction of the overall DM density of the Universe because annihilations of this DM type to the mediators in the early Universe cannot provide the right DM relic density. Models which induce DM self-interactions can alleviate tensions between numerical simulations of collisionless cold DM (CCDM) and astrophysical observations [19-28]. Simulations including DM self-interactions suggest that they have the effect of smoothing out cuspy density profiles, and could solve the problems of CCDM [29-31]. These simulations prefer a self-interaction cross section of $0.1 \mathrm{~cm}^{2} / \mathrm{g} \lesssim \sigma / m \lesssim 10 \mathrm{~cm}^{2} / \mathrm{g}$. There are, however, upper bounds on $\sigma / m$ from a number of sources, including the preservation of ellipticity of spiral galaxies $[32,33]$. The allowed parameter space from these constraints 
nonetheless intersects the range of cross sections which can resolve the small-scale issues of CCDM, in the range $0.1 \mathrm{~cm}^{2} / \mathrm{g} \lesssim \sigma / m \lesssim 1 \mathrm{~cm}^{2} / \mathrm{g}$.

In this Letter, we study repulsive DM-baryon interactions, which allow us to (i) accommodate the decay of neutrons to DM and explain the neutron decay anomaly, (ii) produce NSs with mass over $2 M_{\odot}$, and (iii) account for all of the DM relic density. Our solution to the neutron star stability problem is qualitatively different than those previously proposed using repulsive DM self-interactions [18]. Repulsive self-interactions stiffen the equation of state by raising the DM chemical potential and tuning down the DM to baryon fraction in equilibrium. Repulsive DMbaryon interactions can also stabilize the star, not by stiffening the EOS, but by making it energetically expensive to produce DM particles in a pure baryon medium (and vice versa). Therefore the cross interactions can impede the creation of any significant amount of DM inside the NS, thus maintaining almost pure NSs (without DM present), despite the fact that free neutrons could decay to DM.

Before we present a specific example of a microscopic model that can give all this, it is instructive to show how DM-neutron interactions affect the stability of NSs. We model the interaction by a vector or scalar boson mediated Yukawa potential

$$
U= \pm \frac{g_{\chi} g_{n}}{4 \pi} \frac{e^{-m_{\phi} r}}{r}
$$

where $+(-)$ is for vector (scalar) exchange, $g_{\chi, n}$ are the couplings to DM and neutron, respectively, and $m_{\phi}$ is the mass of the mediator. Couplings with equal (opposite) sign result in repulsion (attraction) for vectors and vice versa for scalars. DM self-interactions have a similar potential with coupling $g_{\chi}^{2}$ in place of $g_{\chi} g_{n}$. None of the models proposed to date that explain the neutron decay anomaly lead to a repulsive cross interaction, with the exception off Model 1 of Ref. [12] that has a photon in the final state (furthermore, the UCNA collaboration has excluded with 97\% CL the visible decay $n \rightarrow \chi+\gamma$ for $937.900 \mathrm{MeV}<m_{\chi}<$ $938.783 \mathrm{MeV}$ [34]). However it couples to the neutron via a magnetic dipole interaction, which can be attractive or repulsive depending on its orientation. We expect that neutrons will occupy equally spin up and down states, and therefore such interactions will average to zero; hence they are not suitable in our case.

Equation of state.-The energy density in a NS with DM and the above interaction is

$$
\varepsilon\left(n_{n}, n_{\chi}\right)=\varepsilon_{\text {nuc }}\left(n_{n}\right)+\varepsilon_{\chi}\left(n_{\chi}\right)+\frac{n_{\chi} n_{n}}{z^{2}},
$$

where $n_{n, \chi}$ are the neutron and DM number densities respectively, $\varepsilon_{\text {nuc }}$ is the energy density due to nuclear interactions, $\varepsilon_{\chi}$ is the energy density of DM, and the last term is the Yukawa energy density due to $n-\chi$ interactions in the mean field approximation where $z \equiv m_{\phi} / \sqrt{\left|g_{\chi} g_{n}\right|}$. The last term in the energy density is valid as long as the star is large compared to the Yukawa screening length, i.e., $R \gg 1 / m_{\phi}$. Notice that the cross interaction depends simply on one parameter $z$. Long range forces are severely constrained and therefore we are going to assume that the mediator has a mass. The DM energy density including DM self-interactions is

$$
\begin{aligned}
\varepsilon_{\chi} & =\frac{m_{\chi}^{4}}{8 \pi^{2}}\left[x \sqrt{1+x^{2}}\left(1+2 x^{2}\right)-\log \left(x+\sqrt{1+x^{2}}\right)\right] \pm \frac{n_{\chi}^{2}}{2 z^{12}}, \\
x & =\frac{\left(3 \pi^{2} n_{\chi}\right)^{1 / 3}}{m_{\chi}}
\end{aligned}
$$

where $z^{\prime} \equiv m_{\phi} / g_{\chi}$ and it is understood that the last term corresponds to DM self-interactions with $+(-)$ sign being repulsive (attractive). The free energy cost at zero temperature associated to creating a DM particle at fixed total number density $n_{\mathrm{F}}=n_{n}+n_{\chi}$ is just the change in internal energy, i.e.,

$\Delta E \equiv \frac{\partial \varepsilon\left(n_{\mathrm{F}}-n_{\chi}, n_{\chi}\right)}{\partial n_{\chi}}=\mu_{\chi}\left(n_{\chi}\right)-\mu_{\mathrm{nuc}}\left(n_{n}\right)+\frac{n_{\mathrm{F}}-2 n_{\chi}}{z^{2}}$,

where $\mu_{i}(i=\chi$, nuc $)$ represent the chemical potentials of $\mathrm{DM}$ and neutrons, respectively. A chemical equilibrium exists when $\Delta E=0$. In a pure neutron environment where no DM is present the energy cost is

$$
\Delta E_{0}=\left.\Delta E\right|_{n_{\chi}=0}=m_{\chi}-\mu_{\mathrm{nuc}}\left(n_{\mathrm{F}}\right)+\frac{n_{\mathrm{F}}}{z^{2}} .
$$

Notice, that the nuclear chemical potential $\mu_{\text {nuc }}>m_{n}$ and $m_{n}>m_{\chi}$ for the dark decay to take place. Therefore, in the absence of DM self-interactions, weak DM-neutron interactions (large $z$ ) makes neutron conversion thermodynamically favored [15-17], whereas stronger DM-neutron cross interactions (small $z$ ) lead to a large energy cost for converting neutrons to DM that makes it energetically favored to have zero DM density. For the nuclear EOS, we have chosen the SLy-4 [35] which is a nuclear EOS without a quark core and the power law EOS $V_{3 \pi}+V_{\mathrm{R}}$ [36] (which was also used in Ref. [18]). Both EOSs we are using can in isolation support NSs with a mass larger than $2 M_{\odot}$, and hence are consistent with observational data. As depicted in Fig. 1, for a given strength of DM-neutron cross interaction (i.e., for a given $z$ ), there are three possibilities. (i) $\Delta E_{0}>0$; i.e., the system is in a pure neutron phase simply because there is an energy cost to create DM. As can be seen by inspection of Eq. (5), by strengthening cross interactions (i.e., by reducing $z$ ), the system can always enter the $\Delta E_{0}>0$ regime. This is also shown graphically in 


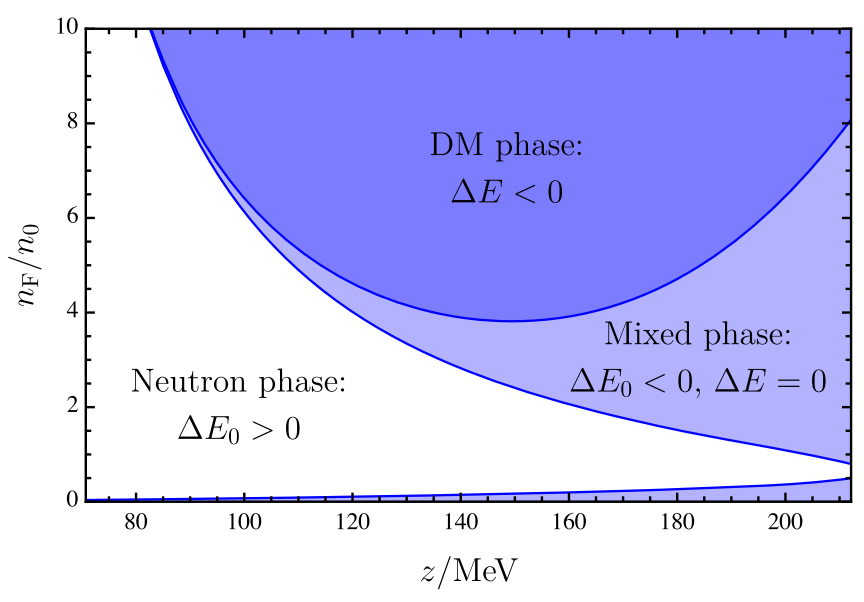

FIG. 1. The phase diagram using Eq. (4) and the SLy-4 EOS [35] assuming $m_{n}-m_{\chi}=1 \mathrm{MeV}$. In the white region there is an energy cost associated to creating any DM particles and pure neutron matter is preferred. In the light blue region, a chemical equilibrium exists with both neutron matter and DM. In the dark blue region, pure DM is thermodynamically preferred. For $z \lesssim 100 \mathrm{MeV}$, fermion densities $<6 n_{0}$ favor neutron matter and therefore do not affect NSs with mass smaller than $2 M_{\odot}$.

Fig. 1, where for a given total density $n_{\mathrm{F}}$, there is always a $z$ below which $\Delta E_{0}>0$ and no DM particles are present. (ii) $\Delta E_{0}<0$ and $\Delta E=0$ for some value of $n_{\chi}<n_{\mathrm{F}}$. In this case $\Delta E_{0}<0$ means that it is energetically favored to convert some of the neutrons to DM. The condition $\Delta E=0$ ensures chemical equilibrium and by enforcing this condition using Eq. (4), one can determine the amount of DM present (i.e., $n_{\chi}$ ). (iii) The last regime satisfies $\Delta E<0$ for any $n_{\chi}$, which means that effectively all neutrons have converted to DM particles. We measure number densities in units of the nuclear density $n_{0}=0.16 \mathrm{fm}^{-3}$. We will mainly focus on DM-baryon cross interaction as the mechanism allowing NSs to reach $2 M_{\odot}$. However, we will also comment on the case where DM self-interactions provide the needed support for heavy NSs.

In order to estimate how heavy NSs can be, we use Eq. (2) with our two choices of nuclear EOS $[35,36]$. The pressure is derived from the relation $P=n_{\mathrm{F}}^{2} d\left(\varepsilon / n_{\mathrm{F}}\right) / d n_{\mathrm{F}}$. In the limit of zero temperature (an excellent approximation for NSs), knowing the pressure and the energy density as a function of $n_{\chi}, n_{\text {nuc }}$ and the parameter $z$, allows us to solve the relativistic hydrostatic equilibrium described by the TolmannOppenheimer-Volkoff equation. By scanning the central fermion density, we find the maximum mass for the NS. Recall that $n_{\chi}$ is uniquely fixed from the chemical equilibrium condition $\Delta E=0$ of Eq. (4). If $\Delta E_{0}>0$, we obviously have $n_{\chi}=0$. Therefore NSs can be as heavy as $2 M_{\odot}$ based on the fact that no DM is present to soften the EOS (corresponding to a central density of $6 n_{0}$ using the SLy-4 EOS shown in Fig. 1). For our choices of EOS this corresponds to $z \lesssim 100 \mathrm{MeV}$. No stable equilibrium exists if the central density is in the pure DM phase, and the heaviest stable configuration in the mixed phase is around $1.5 M_{\odot}$.

A microscopic model.-We move on to suggest a concrete model satisfying the two basic assumptions, i.e., to provide the mechanism for the neutron decay to DM and provide repulsive cross interactions with $z \lesssim 100 \mathrm{MeV}$. The potential in Eq. (1) can arise from scalar or vector exchange. In the former case the repulsive force requires opposite sign charges of $\chi$ and $n$, while in the latter same sign charges are required. In this example, we choose a scalar mediator, and realize the model as a modification of Model 2 of Ref. [12]. It requires four particles beyond the Standard Model (SM): a scalar $\Phi=$ $(3,1)_{-1 / 3}$ (color triplet, weak singlet, hypercharge $-1 / 3$ ), two Dirac fermions $\tilde{\chi}$ and $\chi$ (the DM particle), and a scalar $\phi$ (all SM singlets). The neutron decay to $\chi+\phi$ is mediated by the very heavy $\Phi$ and the fermion $\tilde{\chi}$ with a mass that may be heavier than the neutron, through interactions given by

$$
\begin{aligned}
\mathcal{L}= & \lambda_{q} \epsilon^{i j k} \overline{u_{L i}^{c}} d_{R j} \Phi_{k}+\lambda_{\chi} \Phi^{* i} \overline{\tilde{\chi}} d_{R i}+\lambda_{\phi} \overline{\tilde{\chi}} \chi \phi \\
& +\mu H^{\dagger} H \phi+g_{\chi} \bar{\chi} \chi \phi+\text { H.c. },
\end{aligned}
$$

where $d_{R}$ and $u_{R}$ are the standard model singlet quarks of charge $-1 / 3$ and $2 / 3$. The dark neutron decay takes place with interactions in the first line of the Lagrangian. The baryon numbers for the particles $\Phi, \tilde{\chi}, \chi$ and $\phi$ are chosen to be $-2 / 3,1,1$, and 0 , respectively. The model requires a fine tuning if one insists on $m_{\phi} \ll m_{\chi}$; this is also the case in the original Model 2 of Ref. [12]. One can envision alternative scenarios where, e.g., the mediator might be a Goldstone boson with a mass protected from big corrections. However this goes beyond the scope of this Letter, since we intend to provide the simplest possibility here. Compared to Model 2 in Ref. [12] the baryon numbers of $\chi$ and $\phi$ are exchanged which allows additional terms in the Lagrangian, in particular a Higgs portal and a vertex with $\chi$ and $\phi$. At low energies, the interaction through the Higgs portal induces an effective interaction with the neutron $g_{n} \phi \bar{n} n$ where

$$
g_{n}=\frac{\mu \sigma_{N}}{m_{h}^{2}},
$$

where $m_{h}=125 \mathrm{GeV}$ is the Higgs mass and $\sigma_{N} \equiv \sum_{\text {all }}\left\langle n\left|m_{q} \bar{q} q\right| n\right\rangle \approx 280 \mathrm{MeV}$, using $\sigma_{N}=1 / 9\left(2 m_{n}+\right.$ $\left.7 \sigma_{u d s N}\right)$ [37] and $\sigma_{u d s N} \equiv \sum_{q=u, d, s}\left\langle n\left|m_{q} \bar{q} q\right| n\right\rangle=86.1(20.1)$ from Ref. [38]. The model can incorporate the neutron decay to DM and DM relic density just as described in Ref. [12]. The repulsive DM-neutron interactions require $g_{n} g_{\chi}<0$. To get the right interaction strength $z \lesssim 100 \mathrm{MeV}$, we must consider constraints on the light mediator $\phi$. First we consider constraints on the DM self-interaction coupling $g_{\chi}$, which allows a DM scattering cross section per mass $\sigma / m \lesssim 1-10 \mathrm{~cm}^{2} / \mathrm{g}$, with the relevant momentum transfer weighted cross section given by [39] 


$$
\sigma_{\mathrm{T}}=\frac{4 \pi}{m_{\phi}^{2}} \beta^{2} \log \left(1+\beta^{-1}\right),
$$

with $\beta=2 \alpha m_{\phi} /\left(m_{\chi} v^{2}\right)$ and $v \sim 30 \mathrm{~km} / \mathrm{sec}$ for typical dwarf galaxies (the expression being valid for $\beta<0.1$ and $\left.m_{\chi} v / m_{\phi} \gg 1\right)$. Taking the DM mass to be $\sim m_{n}$, we get $g_{\chi} \lesssim 4 \times 10^{-4}$ with a mild dependence on the mediator mass $m_{\phi}$. For a $g_{\chi}$ value slightly smaller than $4 \times 10^{-4}$, the DM self-interactions fall in the range that alleviate the problems of CCDM. At the same time, the constraints on light particles coupling to the neutron allow us to find parameter space satisfying our NS stability condition. We can for example choose $m_{\phi} \sim 0.1 \mathrm{eV}$ and $g_{n} \sim-10^{-14}$, corresponding to $\mu \sim-0.6 \mathrm{eV}$. Such a value of $g_{n}$ is allowed by the strict constraints set on arguments of rapid red giant star cooling, (see, e.g., Fig. 3 in Ref. [40]). These values correspond to $z \sim 50 \mathrm{MeV}$, which is below the value $100 \mathrm{MeV}$ that we found sufficient to stabilize heavy neutron stars. We should stress here that close to the surface of the NS where $n_{F} \rightarrow 0$, one cannot exclude the presence of DM, simply because at low densities $\mu_{n} \simeq m_{n}$ while $\mu_{\chi} \simeq m_{\chi}$. Since $m_{n}>m_{\chi}$, close to the surface there could be neutrons converting to DM. This can be seen at low densities in our Fig. 1. However, this does not change our conclusions. First, a small amount of DM at low densities close to the surface does not change the overall stability of the star which depends on the EOS at the center. Second, at the NS crust there are heavy nuclei, and the EOSs we have been using are not accurate.

The light mediator $\phi$ could potentially create problems during $\mathrm{BBN}$ and $\mathrm{CMB}$ since it could contribute to the effective number of relativistic degrees of freedom. We require that $\phi$ decays before the start of $\mathrm{BBN}$ in order not to disturb the abundances of light elements. This can be achieved by decaying to active or sterile neutrinos as, e.g., in Ref. [41]. In the case of sterile neutrinos, the decay can take place via a term $y_{N} \phi N^{c} N$ where $N$ is a light sterile neutrino. The requirement that $\phi$ decays before $\mathrm{BBN}$ leads to the condition $y_{N}>2 \times 10^{-7}\left(0.1 \mathrm{eV} / m_{\phi}\right)^{1 / 2}$. Alternatively, $\phi$ can decay to active neutrinos via an effective Weinberg operator of the form $\phi(L H)^{2} / \Lambda^{2}$ as long as the scale $\Lambda \lesssim 6 \times 10^{6} \sqrt{m_{\phi} / 0.1 \mathrm{eV}} \mathrm{GeV}$. This induces an effective coupling between $\phi$ and active neutrinos of the order or larger than $v_{\mathrm{EW}}^{2} / \Lambda^{2}=1.7 \times 10^{-9}$. Neutrino dump experiments can in principle set constraints on couplings of neutrinos to other light particles (see, e.g., Ref. [42] for a review).

Self-interactions.-A qualitatively different way of accommodating dark neutron decays in NSs with masses above $2 M_{\odot}$ is via DM self-interactions. In the case of repulsive DM-baryon interactions, the existence of DM inside the star is disfavored. In the case of DM selfinteractions, DM is present but has a relatively stiff EOS. As was confirmed also in Ref. [18], in order to support heavy NSs via DM self-interactions, a light vector mediator is needed. If DM is produced thermally, the relic density must be determined by the DM annihilations to these mediators. However, the mediators themselves have to decay to SM particles before the BBN epoch. This sets a minimum coupling between the mediator and SM, which is sufficient to deplete most of the DM relic density. Therefore in such a setup, the DM particle that the neutron decays to cannot account for the whole relic density of DM. This outcome can however be avoided if DM is created with a matter-antimatter asymmetry. The $t$-channel annihilation rate of DM fermions into light vector mediators is $\sigma_{\text {ann }} v=$ $g_{\chi}^{4} /\left(16 \pi m_{\chi}^{2}\right)\left(1-m_{\phi}^{2} / m_{\chi}^{2}\right)^{1 / 2}$ [43], which can be much larger than the typical weak scale annihilation rate for couplings larger than $g_{\chi} \sim 2 \times 10^{-2}$. In this case the whole population of anti-DM is annihilated. Moreover, it has been argued that a primordial DM asymmetry can induce lowscale baryogenesis through resonant dark matter-neutron oscillations in the early universe [44]. Furthermore, asymmetric DM immediately avoids the bounds set in large volume Cerenkov detectors from the absence of anti-DM annihilation with nuclei [45]. For a coupling $g_{\chi}>2 \times 10^{-2}$, the self-interaction cross section becomes $\sigma / m \lesssim 10 \mathrm{~cm}^{2} / \mathrm{g}$ for $z^{\prime} \gtrsim 36 \mathrm{MeV}$, corresponding to a mediator mass larger than $m_{\phi} \gtrsim 0.7 \mathrm{MeV}$. If the mediator mass furthermore exceeds $2 m_{e}$ the decay channel to $e^{+} e^{-}$opens up and it is easy to accommodate the particle's decay before BBN (see Fig. 2 of Ref. [18]).Therefore as long as these constraints are satisfied and $g_{\chi}$ is much larger than $2 \times 10^{-2}$, asymmetric DM models would be able to account for the whole DM relic density. However, as can be seen from Fig. 2, in order to have NSs with mass over $2 M_{\odot}$, $z^{\prime}=m_{\phi} / g_{\chi}$ must be smaller than a given value. For example the SLy-4 EOS requires $z^{\prime} \lesssim 25 \mathrm{MeV}$, whereas $V_{3 \pi}+V_{R}$

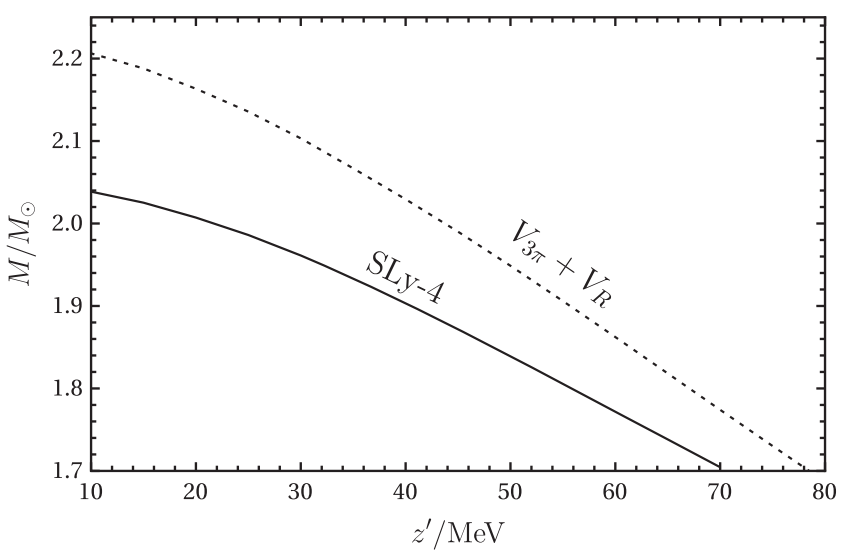

FIG. 2. The maximum NS mass with DM self-interactions as a function of interaction parameter $z^{\prime}$. The figure shows two different EOS; SLy-4 is relatively soft and $M \geq 2 M_{\odot}$ requires $z^{\prime} \lesssim 25 \mathrm{MeV}$, whereas $V_{3 \pi}+V_{R}$ requires that $z^{\prime} \lesssim 45 \mathrm{MeV}$. Both cases assume that the mass of DM is one $\mathrm{MeV}$ below that of the neutron. 
requires $z^{\prime} \lesssim 45 \mathrm{MeV}$. Given the conditions we stated above, i.e., having a $g_{\chi} \gg 2 \times 10^{-2}$ and satisfying the DM self-interactions constraints $\left(z^{\prime} \gtrsim 36 \mathrm{MeV}\right)$, one can see that $2 M_{\odot}$ NSs with allowed DM self-interactions and a SLy-4 EOS cannot be achieved, whereas it will be marginally possible for $V_{3 \pi}+V_{R}$. For an ultrahard EOS where $z^{\prime}$ can even be as large as $60 \mathrm{MeV}$ [18], DM self-interactions can accommodate easier the maximum mass of $2 M_{\odot}$. The DM-baryon cross interactions presented here provide a appealing solution to the problem because there is neither a need for strong self-interactions nor an asymmetric DM production mechanism.

Conclusions. - In case where the neutron decay discrepancies are due to partial neutron decays to DM, we have shown that a repulsive interaction between DM and neutrons can disfavor the conversion of neutrons to DM inside NSs, thus allowing NSs to be heavier than $2 M_{\odot}$, as supported by observations. We propose a microscopic model that can accommodate the DM-baryon cross interactions relying on a light scalar and neutron interactions via a Higgs portal. This is qualitatively different from previous proposals because in our scenario, it is the absence of DM that saves heavy NS from collapsing.

C. K. is grateful to Mark Alford and Steven Harris for valuable comments on EOS. The work of B.G. was supported in part by the DOE Grant No. DESC0009919. C. K. is partially funded by the Danish National Research Foundation, Grant No. DNRF90, and by the Danish Council for Independent Research, Grant No. DFF 4181-00055.

*bgrinstein@ucsd.edu

†kouvaris@cp3.sdu.dk

\#ngnielsen@cp3.sdu.dk

[1] C. Patrignani et al. (Particle Data Group), Chin. Phys. C 40, 100001 (2016).

[2] W. Mampe, L. N. Bondarenko, V. I. Morozov, Y. N. Panin, and A. I. Fomin, Pis'ma Zh. Eksp. Teor. Fiz. 57, 77 (1993) [JETP Lett. 57, 82 (1993)].

[3] A. Serebrov et al., Phys. Lett. B 605, 72 (2005).

[4] A. Pichlmaier, V. Varlamov, K. Schreckenbach, and P. Geltenbort, Phys. Lett. B 693, 221 (2010).

[5] A. Steyerl, J. M. Pendlebury, C. Kaufman, S. S. Malik, and A. M. Desai, Phys. Rev. C 85, 065503 (2012).

[6] S. Arzumanov, L. Bondarenko, S. Chernyavsky, P. Geltenbort, V. Morozov, V. V. Nesvizhevsky, Y. Panin, and A. Strepetov, Phys. Lett. B 745, 79 (2015).

[7] J. Byrne and P. G. Dawber, Europhys. Lett. 33, 187 (1996).

[8] A. T. Yue, M. S. Dewey, D. M. Gilliam, G. L. Greene, A. B. Laptev, J. S. Nico, W. M. Snow, and F. E. Wietfeldt, Phys. Rev. Lett. 111, 222501 (2013).

[9] S. Gardner and B. Plaster, Phys. Rev. C 87, 065504 (2013).

[10] C. C. Chang et al., Nature (London) 558, 91 (2018).
[11] A. Czarnecki, W. J. Marciano, and A. Sirlin, Phys. Rev. Lett. 120, 202002 (2018).

[12] B. Fornal and B. Grinstein, Phys. Rev. Lett. 120, 191801 (2018).

[13] M. Pfützner and K. Riisager, Phys. Rev. C 97, 042501(R) (2018).

[14] K. Riisager et al., Phys. Lett. B 732, 305 (2014).

[15] D. McKeen, A. E. Nelson, S. Reddy, and D. Zhou, Phys. Rev. Lett. 121, 061802 (2018).

[16] G. Baym, D. H. Beck, P. Geltenbort, and J. Shelton, Phys. Rev. Lett. 121, 061801 (2018).

[17] T. F. Motta, P. A. M. Guichon, and A. W. Thomas, J. Phys. G 45, 05LT01 (2018).

[18] J. M. Cline and J. M. Cornell, J. High Energy Phys. 07 (2018) 081.

[19] B. Moore, Nature (London) 370, 629 (1994).

[20] R. A. Flores and J. R. Primack, Astrophys. J. 427, L1 (1994).

[21] J. F. Navarro, C. S. Frenk, and S. D. M. White, Astrophys. J. 490, 493 (1997).

[22] A. A. Klypin, A. V. Kravtsov, O. Valenzuela, and F. Prada, Astrophys. J. 522, 82 (1999).

[23] B. Moore, S. Ghigna, F. Governato, G. Lake, T. R. Quinn, J. Stadel, and P. Tozzi, Astrophys. J. 524, L19 (1999).

[24] G. Kauffmann, S. D. M. White, and B. Guiderdoni, Mon. Not. R. Astron. Soc. 264, 201 (1993).

[25] L. Liu, B. F. Gerke, R. H. Wechsler, P. S. Behroozi, and M. T. Busha, Astrophys. J. 733, 62 (2011).

[26] E. J. Tollerud, M. Boylan-Kolchin, E. J. Barton, J. S. Bullock, and C. Q. Trinh, Astrophys. J. 738, 102 (2011).

[27] L. E. Strigari and R. H. Wechsler, Astrophys. J. 749, 75 (2012).

[28] M. Boylan-Kolchin, J. S. Bullock, and M. Kaplinghat, Mon. Not. R. Astron. Soc. 415, L40 (2011).

[29] R. Dave, D. N. Spergel, P. J. Steinhardt, and B. D. Wandelt, Astrophys. J. 547, 574 (2001).

[30] M. Vogelsberger, S. Genel, D. Sijacki, P. Torrey, V. Springel, and L. Hernquist, Mon. Not. R. Astron. Soc. 436, 3031 (2013).

[31] M. Rocha, A. H. G. Peter, J. S. Bullock, M. Kaplinghat, S. Garrison-Kimmel, J. Onorbe, and L. A. Moustakas, Mon. Not. R. Astron. Soc. 430, 81 (2013).

[32] J. L. Feng, M. Kaplinghat, H. Tu, and H. B. Yu, J. Cosmol. Astropart. Phys. 07 (2009) 004.

[33] J. L. Feng, M. Kaplinghat, and H. B. Yu, Phys. Rev. Lett. 104, 151301 (2010)

[34] Z. Tang et al., Phys. Rev. Lett. 121, 022505 (2018).

[35] F. Douchin and P. Haensel, Astron. Astrophys. 380, 151 (2001).

[36] S. Gandolfi, J. Carlson, and S. Reddy, Phys. Rev. C 85, 032801(R) (2012).

[37] M. A. Shifman, A. I. Vainshtein, and V. I. Zakharov, Phys. Lett. 78B, 443 (1978).

[38] Y. B. Yang, A. Alexandru, T. Draper, J. Liang, and K.-F. Liu (xQCD Collaboration), Phys. Rev. D 94, 054503 (2016).

[39] S. Tulin, H. B. Yu, and K. M. Zurek, Phys. Rev. Lett. 110, 111301 (2013). 
[40] J. Heeck, Phys. Lett. B 739, 256 (2014).

[41] C. Kouvaris, I. M. Shoemaker, and K. Tuominen, Phys. Rev. D 91, 043519 (2015).

[42] M. Bauer, P. Foldenauer, and J. Jaeckel, J. High Energy Phys. 07 (2018) 094.
[43] S. Tulin, H. B. Yu, and K. M. Zurek, Phys. Rev. D 87, 115007 (2013).

[44] T. Bringmann, J. M. Cline, and J. M. Cornell, Phys. Rev. D 99, 035024 (2019).

[45] M. Jin and Y. Gao, Phys. Rev. D 98, 075026 (2018). 